\title{
Management of complex palatoradicular groove in maxillary lateral incisor
}

\author{
Dr. Ripu Singh, ${ }^{1}$ Dr. Shivalal Sharma, ${ }^{1}$ Dr. Khushboo Goel ${ }^{1}$ \\ ${ }^{1}$ Department of Periodontology and Oral Implantology, College of Dental Surgery, \\ B.P. Koirala Institute of Health Sciences, Dharan, Nepal
}

\begin{abstract}
Palatoradicular grooves are the developmental anomalies that owing to complexity in morphology and extent along the root length, can be an aetiology for periodontal destruction, endodontic involvement or both. We report a case of a 34 years male patient who presented with a chief complaint of pain and swelling in his upper left front region of jaw since one month. Clinical and radiographic examination confirmed presence of periodontal-endodontic lesion with respect to 22. Endodontic treatment followed by periodontal debridement, regenerative procedures, odontoplasty and sealing of the groove was done. Combined endodontic-periodontal approach was successful in resolving the pathology with satisfactory and stable healing seen clinically and radiographically for upto six months postoperative.

Keywords: Endodontic-periodontal approach; palatoradicular groove; periodontal-endodontic lesion.
\end{abstract}

\section{INTRODUCTION}

Palatoradicular groove (PRG) is a developmental anomaly usually found on the lingual aspect of maxillary incisors in which an infolding of the inner enamel epithelium and Hertwig's epithelial root sheath create a groove that start in the cingulum region, proceeding apically towards and beyond cementoename $\mathrm{e}^{\mathrm{l}}$ junction and may terminate at various levels in the root. ${ }^{1}$ PRG has a reported incidence ranging from 2.8 to $18 \%{ }^{2}$ with higher prevalence in lateral incisors (4.4-5.6\%). ${ }^{3}$ PRG can be classified based on its location as distal, mesial or mild, moderate and complex types according to the depth and complexity of the groove. ${ }^{4}$

\section{CASE REPORT}

A 39 years old male patient reported to the Department of periodontology and oral implantology with the chief complaint of pain and swelling in the upper left front region of jaw since 1 month. Intraoral clinical examination revealed a draining sinus through attached gingival in between 21 and 22 buccally (Figure 1a) and localised circumscribed inflammation of the marginal gingiva palatally with probing pocket depth of $9 \mathrm{~mm}$ (Figure 1b) along a groove on palatal aspect of tooth with respect to 22. Tenderness on vertical

\section{Correspondence:}

Dr. Ripu Singh

Department of Periodontology and Oral Implantology,

College of Dental Surgery, B.P. Koirala Institute of Health

Sciences, Dharan, Nepal

Email: drripusingh2017@gmail.com

\section{Citation}

Singh R, Sharma S, Goel K. Management of complex palatoradicular groove in maxillary lateral incisor. J Nepal Soc Perio Oral Implantol. 2020;4(7):47-9.

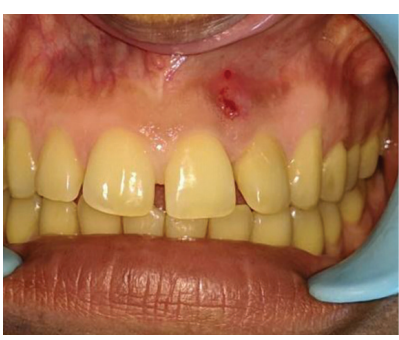

Figure 1a: Buccal view.

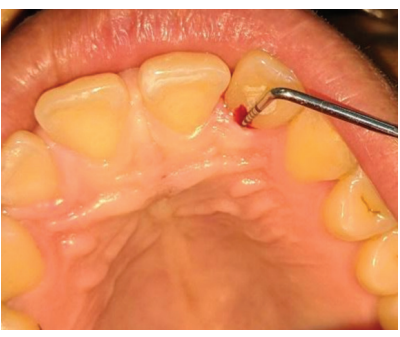

Figure 1b: Probing on palatal aspect with respect to 22 .

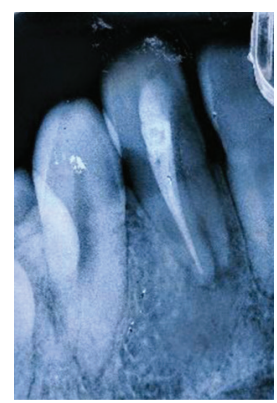

Figure 2: Intraoral periapical radiograph with respect to 22 .

percussion and grade I mobility also was present on the same tooth. Intraoral periapical radiograph (IOPAR) with respect to 22 revealed linear radiolucency extending from the coronal region till the apex and also a linear radicular radio-opacity suggestive of tooth being treated endodontically. In addition, periapical regions showed ill-defined radiolucency (Figure 2).

\section{Management}

Initially nonsurgical therapy (scaling root planing) was completed following which retreatment of root canals in relation to 22 was done with endodontic consultation. After completion of endodontic treatment, mucoperiosteal

DOI: https://doi.org/10.3126/jnspoi.v4i1.30905 


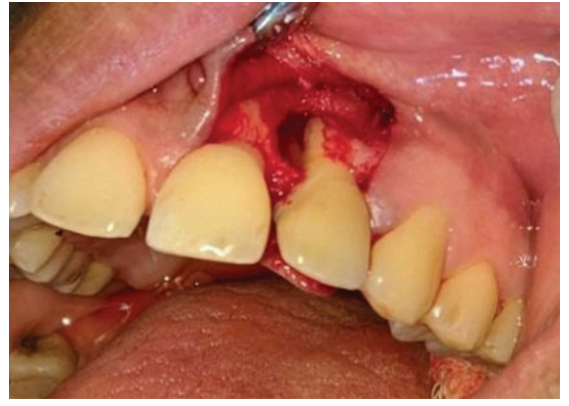

Figure 3: After reflection of mucoperiosteal flap.

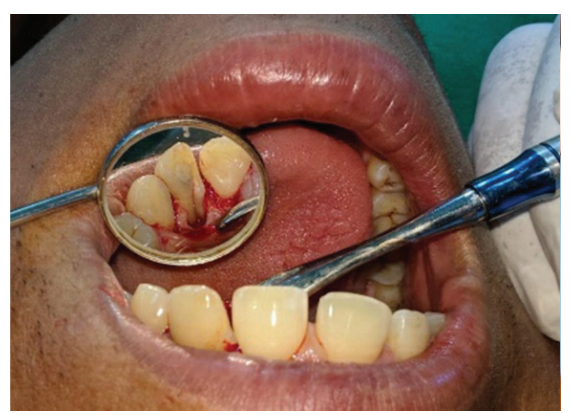

Figure 6: Palatoradiucular groove after debridement.

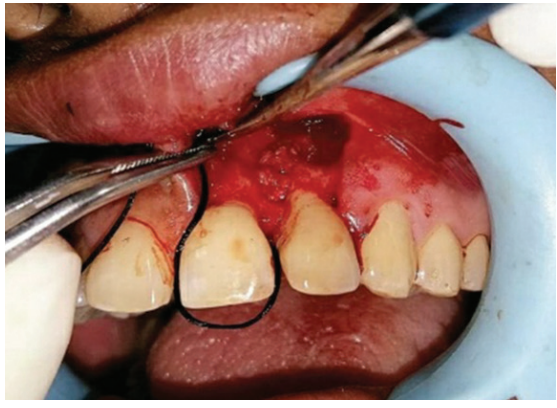

Figure 4: Placement of bone graft and PRF.

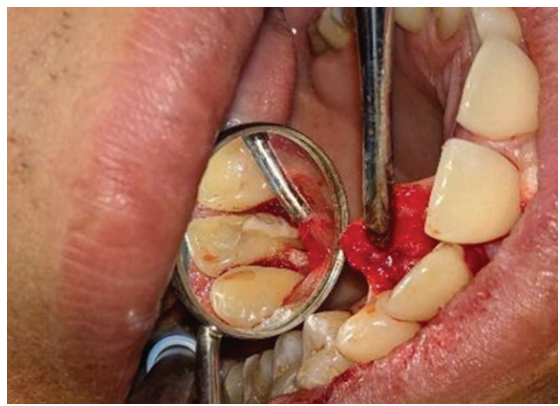

Figure 7: Palatoradiucular groove sealed with

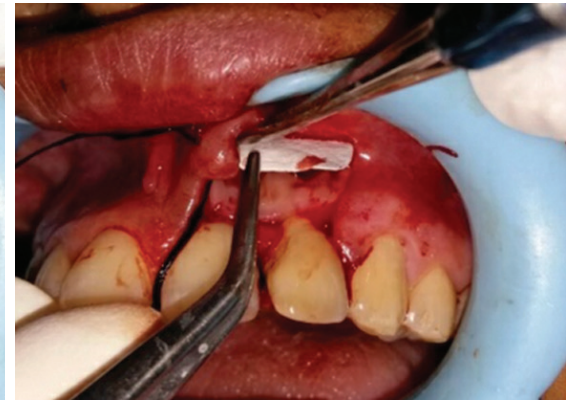

Figure 5: Placement of PRF membrane and barrier membrane.

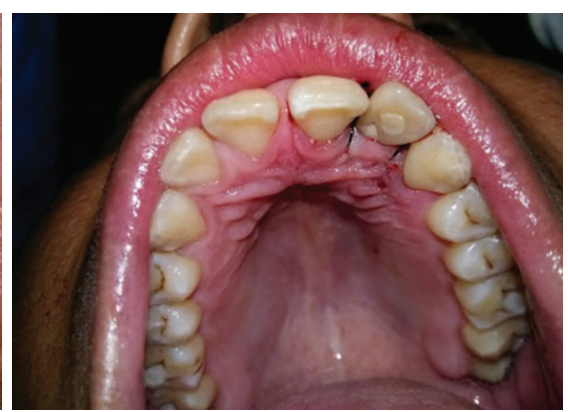

Figure 8: Interrupted sling suturing with 3-0 silk suture.

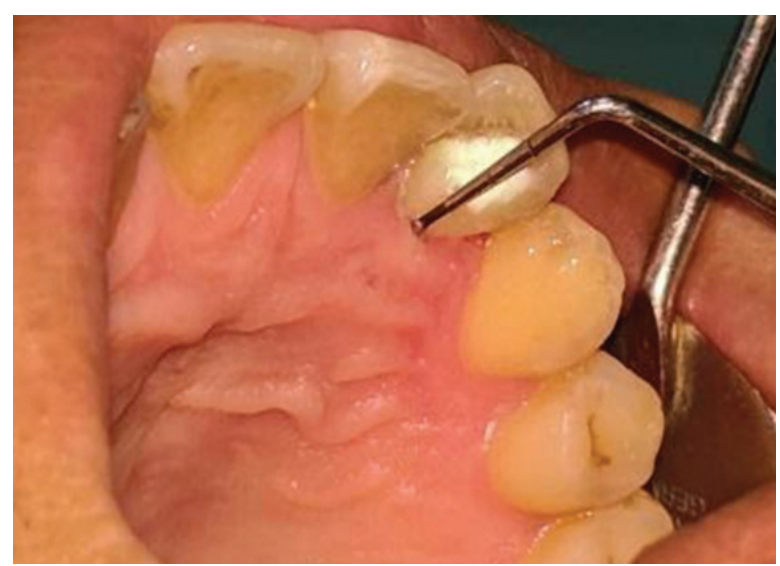

Figure 9: Six months postoperative with probing.

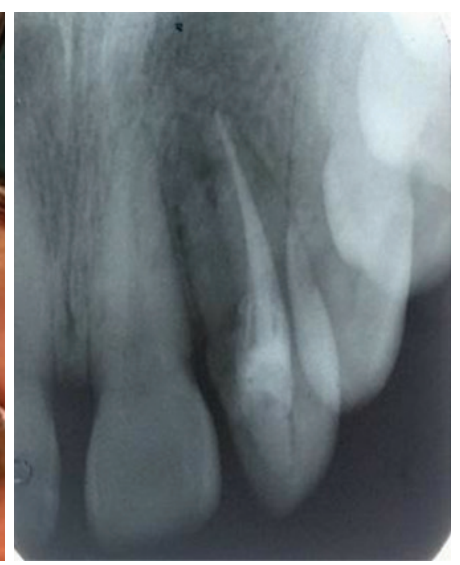

Figure 10: Six months postoperative intraoral periapical radiograph. flap with a releasing incision buccally and an envelope flap on palatal aspect was elevated. Meticulous scaling root planing and debridement of granulation tissue revealed a palatoradicular groove extending beyond the middle third of the root and was deeply invaginated, separating an accessory root from the main root trunk. The root bifurcation area was also debrided meticulously. A combined (two and three walled) osseous defect was present on mesiobuccal aspect and along palatoradicular groove. Regenerative procedure was carried out using bone graft ( $\beta$-tricalcium phosphate and hydroxyapatite), platelet rich fibrin (PRF), resorbable barrier membrane and PRF membrane (Figure 4 and 5). The palatoradicular groove was sealed properly with glass ionomer cement. Subsequently, the palatal bone defect along the PRG that measured $11 \mathrm{~mm}$ depth and $2 \times 3 \mathrm{~mm} 2$ in width at the crest was filled using bone graft (alloplast) and covered with resorbable barrier membrane. Flap closure was obtained with simple-loop (interrupted) suture using 3-0 silk suture (Figure 8 and 9).

\section{Postoperative management and outcomes}

Ibuprofen $400 \mathrm{mg}$ twice daily for two days was given. Amoxicillin $500 \mathrm{mg}$ thrice daily for seven days and chlorhexidine mouthrinse $0.2 \%$ twice daily for two weeks were prescribed. Suture removal was done on the tenth day and self-performed oral hygiene measures were reinforced. The patient was then kept under monthly recall for 4 months. At one month postoperative visit, uneventful healing was observed and the patient was asymptomatic. At three months, probing depth on the palatal aspect measured $3 \mathrm{~mm}$ which continued to remain at the same level even at six months (Figure 9). 


\section{DISCUSSION}

Morphological features of PRG compromises patient's selfcare and favours accumulation of plaque, calculus or food debris; where from localised periodontal inflammation can initiate and progress along the tunnel like pathway, precipitating series of consequences like periodontal pocket, attachment loss and bone loss. In addition, secondary endodontic involvement via apical or lateral canals can predispose to the development of complex perio-endo lesions. ${ }^{5}$

Simple/ shallow grooves are less likely to cause severe destruction and can simply be managed by saucerization or odontoplasty. In the contrary, complicated grooves which communicate with the pulp cavity either laterally or apically may necessitate additional measures like restoration of the defect. $^{4}$

In our case, the palatoradicular groove was classified as complex since it was judged to extend more than two-thirds of the root during clinical probing, radiographic examination and later confirmed on reflection of the flap. The groove with adjacent deep narrow bony defect and periapical radiolucency apparently rendered a communication between the oral environment and pulp resulting in perio-endo lesion. The groove was sealed to enhance regeneration of periodontal attachment and bone and to eliminate the pocket. Materials such as composite and amalgam have been also used to seal the PRG. ${ }^{6}$ In our case, glass ionomer cement which was chosen as the bonding to the dentin, is better and it also enhances epithelial and connective tissue attachment. ${ }^{7}$ Recently, mineral trioxide aggregate has also been used for this purpose, but it has a disadvantage of getting washed off from the transgingival defect in the presence of moisture. ${ }^{8}$

The health of the periapical osseous tissue appears to be the pivotal factor for tooth retention. ${ }^{4}$ Therefore, by maintaining the health and integrity of the periapical bone or encouraging the periapical bone to regenerate with combined endodontic and periodontal treatment, the approaches to prevent apical ingress of microorganisms may be rendered more likely to succeed.

A combined endodontic and periodontal therapy as an interdisciplinary approach proved to be successful in the tooth with complex palatoradicular groove associated with substantial periapical periodontal breakdown having a poor prognosis for tooth retention. Accurate diagnosis and selection of appropriate treatment modality can help in saving the teeth with such anomalies.

\section{ACKNOWLEDGEMENT}

Dr. Khushboo Goel, Dr. Sajeev Shrestha, and Dr. Pujan Acharya, Department of Periodontology and Oral Implantology, College of Dental Surgery, BPKIHS, Dharan.

Conflict of interest: None.

\section{REFERENCES}

1. Withers JA, Brunsvold MA, Killoy WJ, Rahe AJ. The relationship of palato-gingival grooves to localized periodontal disease. J Periodontol. 1981; 52 (1):41-4. doi:10.1902/jop.1981.52.1.41

2. Hou GL, Tsai CC. Relationship between palato-radicular grooves and localized periodontitis. J Clin Periodontol. 1993;20(9):678-82. doi:10.1111/j.1600-051x.1993.tb00715.x

3. Kogon SL. The prevalence, location and conformation of palato-radicular grooves in maxillary incisors. J Periodontol. 1986; 57(4):231234. doi:10.1902/jop.1986.57.4.231

4. Goon WWY, Carpenter WM, Brace NM, Ahlfeld RJ. Complex facial radicular groove in a maxillary lateral incisor. 1991;17(5):244-8.

5. Kim HJ, Choi Y, Yu MK, Lee KW, Min KS. Recognition and management of palatogingival groove for tooth survival: a literature review. Restor Dent Endod. 2017;42(2):77-86. doi:10.5395/rde.2017.42.2.77

6. Friedman S, Goultschin J. The radicular palatal groove--a therapeutic modality. Endod Dent Traumatol. 1988; 4 (6):282-6. doi:10.1111/j.1600-9657.1988.tb00649.x

7. Dragoo MR. Resin-ionomer and hybrid-ionomer cements: part II, human clinical and histologic wound healing responses in specific periodontal lesions. Int J Periodontics Restorative Dent. 1997;17(1):75-87.

8. Attam K., Tiwary R., Talwar S., Lamba A. K. Palatogingival groove: endodontic-periodontal management - case report. Journal of Endodontia. 2010; 36(10):1717-20. doi:0.1016/j.joen.2010.06.025. 